\title{
浓差电池法用于铝液脱测氢的研究
}

\author{
王 东 ${ }^{1}$, 刘春明 ${ }^{2}$, 李胜利 ${ }^{1}$, 李维娟 ${ }^{1}$, 陈树江 ${ }^{1}$, 杨博威 ${ }^{1}$
}

(1. 辽宁科技大学 材料与冶金学院, 鞍山 114051; 2. 东北大学 材料科学与工程学院, 沈阳 110819)

摘 要: 利用固相反应法制备了纯度较高、粒度较小的 $\mathrm{CaZr}_{0.9} \mathrm{In}_{0.1} \mathrm{O}_{3-\alpha}$ 质子导体管, 将其作为电解质组装成浓差电池 型氢洜和氢传感器, 并对 $760^{\circ} \mathrm{C}$ 铝液进行了脱氢过程和氢含量的测定, 研究了氢传感器的探头组装方式、参比气体 流量和压力等对电动势曲线和阻抗谱的影响, 以及氢泉在改善物理条件下的脱氢效果。结果表明: 倒置式探头的电 动势曲线变化较平滑, 约经 $13 \mathrm{~min}$ 达到较稳定状态, 其传感性能优于正置式探头; 参比气体的流量或压力增加时, 电动势也将随之迅速增大，其原因与电动势受 Nernst 方程控制有关，反之则减小。同时，参比气体流量的增加，会 延长电动势达到平衡所需的时间, 并使电极/电解质界面的电荷转移电阻降低。因此, 为了获得快速、准确的测氢 结果, 组装传感器时探头应倒置, 并根据气体管路特点, 确定合适的参比气体流量并对其进行精确控制。此外, 实 验证明了浓差电池型氢泉在铝液脱氢方面具有可行性和实用价值，有深入研究的必要。

关 键 词: 质子导体; 参比气体; 浓差电池; 氢泉; 氢传感器; 阻抗谱

中图分类号: TB321 文献标识码: A

\section{Determination of Hydrogen Content and Dehydrogenation in Molten Aluminum by Concentration Cell Method}

\begin{abstract}
WANG Dong ${ }^{1}$, LIU Chun-Ming ${ }^{2}$, LI Sheng-Li ${ }^{1}$, LI Wei-Juan ${ }^{1}$, CHEN Shu-Jiang ${ }^{1}$, YANG Bo-Wei ${ }^{1}$
(1. School of Materials and Metallurgy, University of Science and Technology, Anshan 114051, China; 2. School of Metallurgy, Northeastern University, Shenyang 110819, China)

Abstract: $\mathrm{The}_{\mathrm{CaZr}} \mathrm{In}_{0.1} \mathrm{O}_{3-\alpha}$ proton conductor was prepared by solid reaction method with high purity and small particle size. Two types hydrogen sensors and pump based on the proton conductor were fabricated for monitoring the hydrogen content and dehydrogenation in molten aluminum at $760^{\circ} \mathrm{C}$. The electromotive force (EMF) and time of hydrogen sensor with the inverted-U electrolyte tube followed a smooth curve in which the EMF tended to be stable after about 13 min whose sensing performance was superior to that with the upright-U electrolyte tube. The sensor EMF increased with the pressure or flow rate of referent gas according to Nernst equation. The equilibrium time of EMF increased and the transferring resistance between the Pt electrode and proton conductor's transfer resistance decreased with the flow rate of reference gas. Therefore, inverted-U type tube and flow rate value of reference gas were very important to acquire the sensitive and accurate hydrogen content values in molten aluminum. In addition, hydrogen pump based on concentration cell had feasibility and actual using value for the dehydrogenation of molten aluminum.
\end{abstract}

Key words: proton conductor; reference gas; concentration cell; hydrogen pump; hydrogen sensor; impedance spectrum 
自 1980 年, Iwahara 等 ${ }^{[1]}$ 首次报道 $\mathrm{Yb}^{3+}$ 掺杂的 $\mathrm{SrCeO}_{3}$ 在高温含氢或含 $\mathrm{H}_{2} \mathrm{O}$ 气氛下具有质子传导 性以来, $\mathrm{ABO}_{3}$ 型 $\left(\mathrm{A}\right.$ 代表 +2 价阳离子, 如 $\mathrm{Sr}^{2+}, \mathrm{Ca}^{2+}$, $\mathrm{Sr}^{2+}, \mathrm{Ba}^{2+}$ 等; $\mathrm{B}$ 代表 +4 价阳离子, 如 $\mathrm{Ce}^{4+}, \mathrm{Ti}^{4+}, \mathrm{Zr}^{4+}$ 等)高温质子导体受到极大关注, 其合成方法、微观 结构和物化性质(如电导率和化学稳定性)等得到广 泛且深入研究 ${ }^{[2-8]}$ 。它在氢百、固体氧化物燃料电池、 膜反应器和水蒸气电解等方面也显示出广阔的应用 前景 ${ }^{[9-13]}$, 且已实现工业化应用。例如, 日本 TYK 公司 ${ }^{[14]}$ 已经开始销售利用 $\mathrm{CaZr}_{0.9} \mathrm{In}_{0.1} \mathrm{O}_{3-\alpha}$ 质子导体 作电解质, $1 \% \mathrm{H}_{2}+\mathrm{Ar}$ 标准气体作参比电极的浓差电 池型传感器, 用于实现对熔铸车间中铝液氢含量的 测定。

采用标准气体作为参比电极的优点在于气体的 性能稳定, 探头的封装也比较方便, 但气体的流量 和压力是否会对测量结果产生影响, 国内外仍未见 明确报道。鉴于此, 本工作采用 $1.06 \% \mathrm{H}_{2}+\mathrm{Ar}$ 标准气 体作为参比电极, 研究参比气体流量和压力等对浓 差电池型传感器测量过程的影响及规律, 并分析其 中的动力学机理。此外, 通过将传感器的两根电极 引线短路的方法, 将传感器改装成氢存尝试进行铝 液电化学脱氢过程。

\section{1 实验方法}

\section{1 材料制备}

将 $\mathrm{ZrO}_{2}(\geqslant 99.9 \mathrm{wt} \%) 、 \mathrm{CaCO}_{3}(\geqslant 99.9 \mathrm{wt} \%)$ 及 $\mathrm{In}_{2} \mathrm{O}_{3}$ $(\geqslant 99.9 \mathrm{wt} \%)$ 三种氧化物粉末按 $\mathrm{CaZr}_{0.9} \mathrm{In}_{0.1} \mathrm{O}_{3-\alpha}$ 的化 学计量比准确称量后放入球磨机中, 以无水乙醇作 介质, $300 \mathrm{r} / \mathrm{min}$ 的转速混磨 $8 \mathrm{~h}$, 然后用小型液压机 以 $20 \mathrm{MPa}$ 的压力将混合粉末压成 $\phi 20 \mathrm{~mm} \times 10 \mathrm{~mm}$ 的圆片, 并在 $1400^{\circ} \mathrm{C}$ 空气气氛下煅烧 $8 \mathrm{~h}$ 。用小型粉 碎机和研钵将煅烧后的圆片制成粉末。XRD 分析表 明, 炦烧后的粉末由 $\mathrm{CaZr}_{0.9} \mathrm{In}_{0.1} \mathrm{O}_{3-\alpha}$ 主相和极微量 的 $\mathrm{CaIn}_{2} \mathrm{O}_{4}$ 相组成(见图 1)。

合成的质子导体粉末用冷等静压机将其压成一 端封闭的 $U$ 型管, 成型压力为 $200 \mathrm{MPa}$, 保压时间 为 $3 \mathrm{~min}$ 。然后在 $1650^{\circ} \mathrm{C}$ 空气气氛中烧结 $10 \mathrm{~h}$, 得 到淡绿色陶瓷化很好的质子导体管, 并以 “ $\cup$ ” (正 置)和 “ $\cap$ ” (倒置)两种形式封接于刚玉管内, 再与 其它相关材料、元件组装成铝液测氢传感器(其结构 参见图 2)。

\section{2 实验方法}

铝液氢含量是通过将测量的铝液温度、电池电

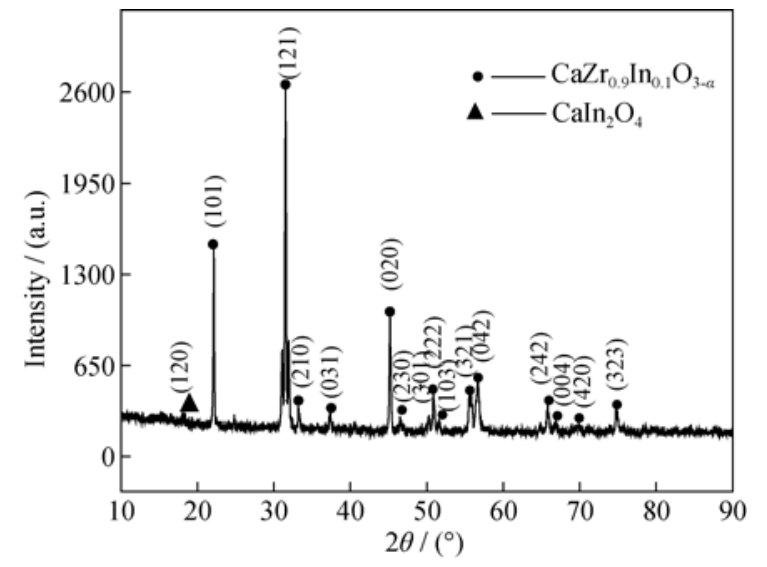

图 1 混合粉末经 $1400^{\circ} \mathrm{C} \times 8 \mathrm{~h}$ 煅烧产物的 XRD 图谱

Fig. 1 XRD pattern of the mixed powders after calcinated at $1400^{\circ} \mathrm{C}$ for $8 \mathrm{~h}$

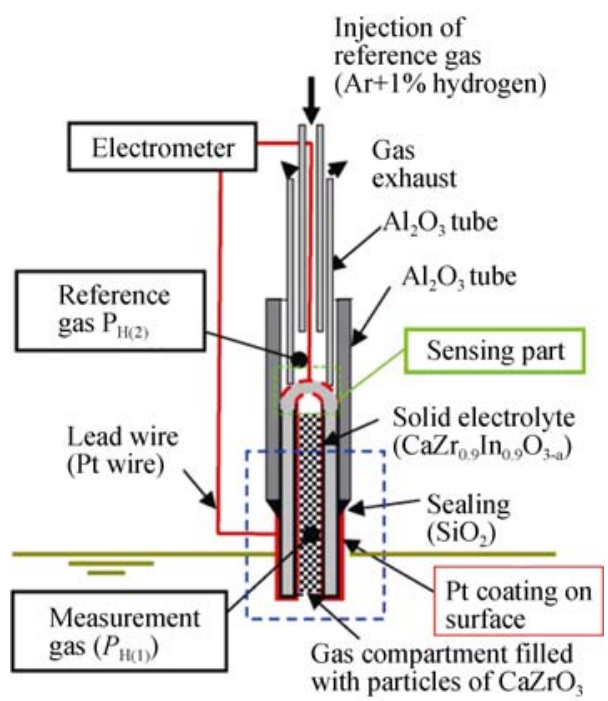

图 2 倒置式氢传感器的结构示意图

Fig. 2 Diagram of structure of hydrogen sensor with invertedU electrolyte tube

动势, 以及已知的参比电极氢分压, 代入由 Nernst 方程和 Sievert 定律推导出的公式中(1)计算出来的。

$$
S=10 \exp [-2760 / T+1.356] \sqrt{P_{\mathrm{H}_{2}} / \exp (2 F E / \mathrm{R} T)}
$$

式中: $P_{\mathrm{H}_{2}}$ 为参比电极氢分压, $\mathrm{mmHg} ; E$ 为电池电动势, $\mathrm{V} ; T$ 为铝液温度, $\mathrm{K} ; \mathrm{R}$ 为理想气体常数, $8.314 \mathrm{~J} /(\mathrm{mol} \cdot \mathrm{K})$; $F$ 为 Faraday 常数, $96500 \mathrm{C} / \mathrm{mol} ; S$ 为铝液中的氢含 量, $\mathrm{mL} / 100 \mathrm{~g} \mathrm{Al}$ 。

实验过程中采用 RIGOL DM3068 6 6 12 位双显数 字万用表测量传感器的电动势(测量点间隔 $4 \mathrm{~s}$, 积 分时间 100PLC); 采用 AUTOLAB PGSTAT30 型电 化学工作站测量阻抗谱(微扰电压 $100 \mathrm{mV}$, 频率范 围 $10^{6} \sim 10^{-2} \mathrm{~Hz}$ ); 采用 ZsimpWin 3.1 软件对阻抗谱进 行拟合处理, 并解析等效电路中各元件的参数意义。 


\section{2 结果与讨论}

\section{1 探头组装方式对电动势的影响}

图 3(a，b)分别是采用倒置式和正置式探头在 $760^{\circ} \mathrm{C}$ 铝液中连续测量时的电动势变化曲线, 由图可 知, 倒置式探头的电动势变化较为平缓, 约经 $13 \mathrm{~min}$ 达到平衡(实验发现此后的电动势也基本保持稳定); 相反, 正置式探头的电动势起伏不平, 难以达到稳 定。这可能是由于正置式探头在铝液中将处于较低 的氧分压下, 此时质子导体具有质子和氧离子的混 合导电状态 ${ }^{[15]}$, 从而影响了正置式探头的准确测量。

\section{2 参比气体压力对电动势的影响}

图 4 为参比气体压力由 $1 \mathrm{kPa}$ 增加到 $2 \mathrm{kPa}$, 待电动 势稳定后又增大到 $3 \mathrm{kPa}$, 再依次减小回 $2 \mathrm{kPa}$ 和 $1 \mathrm{kPa}$ 时电动势的变化曲线。由图 4 可知, 随着参比气体压力 逐渐增大, 传感器的电动势也相应迅速增大, 反之则 减小, 且压力对电动势的影响是一个可逆过程。

由 Nernst 方程(测量时电压表正极连接电解质 管内侧, 见公式(2)) 可知, 当铝液氢分压 $P_{\mathrm{H}_{2}(\mathrm{Al})}$ 恒定 时, 理论电动势 $E$ 随参比气体压力 $P_{\mathrm{H}_{2}(\mathrm{Re})}$ 的增加或 减小而相应的增加或减小。
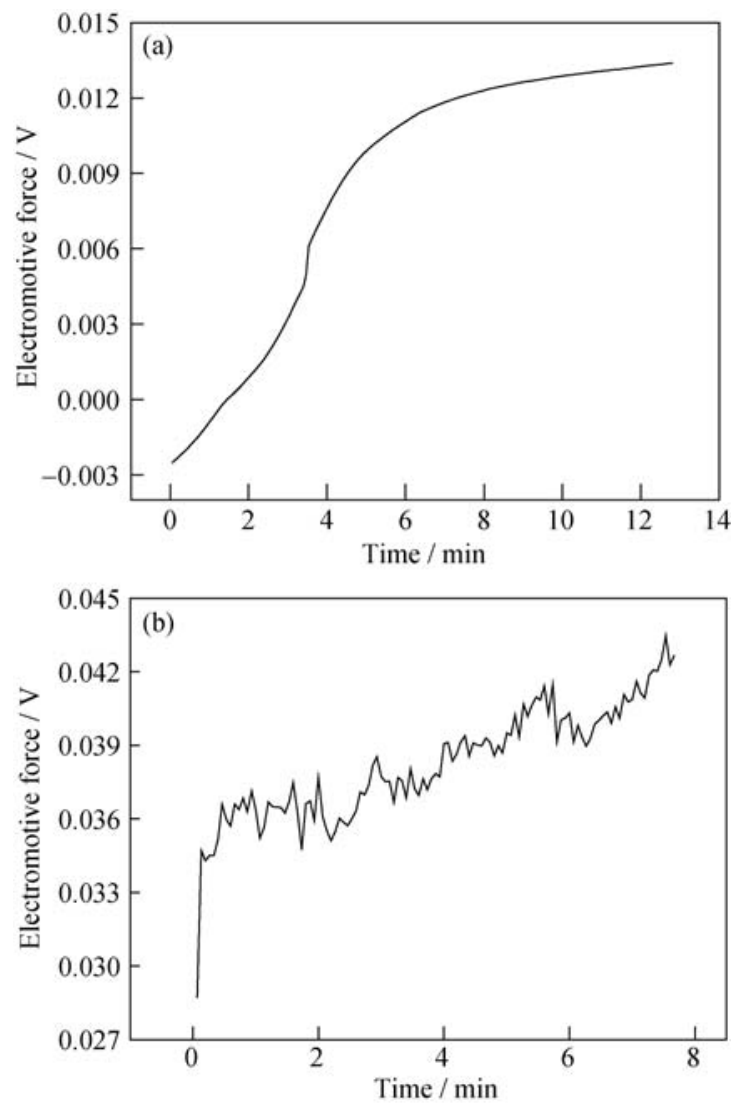

图 3 倒置式(a)和正置式(b)探头的电动势变化

Fig. 3 EMF of hydrogen probe with inverted-U (a) and upright-U (b) electrolyte tube

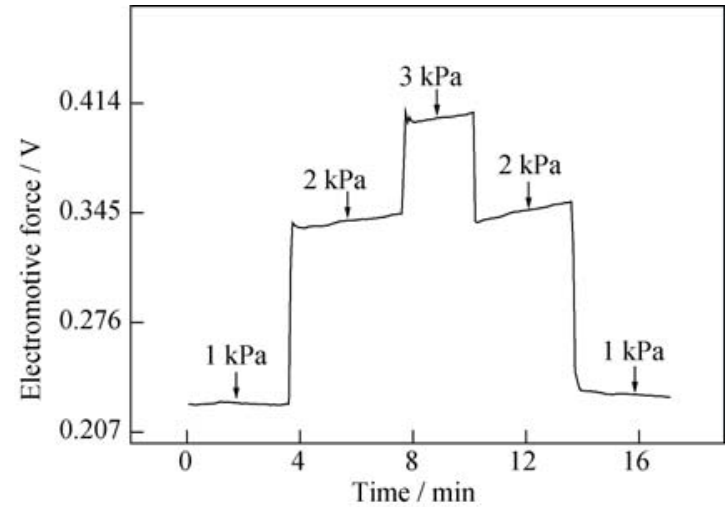

图 4 参比气体压力对传感器电动势的影响

Fig. 4 Dependence of EMF on different pressure of gas

$$
E=\frac{\mathrm{R} T}{2 \mathrm{~F}} \ln \frac{P_{\mathrm{H}_{2}(\mathrm{Re})}}{P_{\mathrm{H}_{2}(\mathrm{Al})}}
$$

式中: $\mathrm{R}$ 为理想气体常数, $8.314 \mathrm{~J} /(\mathrm{mol} \cdot \mathrm{K}) ; T$ 为热力 学温度, $\mathrm{K} ; \mathrm{F}$ 为 Faraday 常数, $96500 \mathrm{C} / \mathrm{mol} ; P_{\mathrm{H}_{2}(\mathrm{Re})}$ 和 $P_{\mathrm{H}_{2}(\mathrm{Al})}$ 分别为参比气体和铝液的氢分压; $E$ 为电 池电动势, $\mathrm{V}$ 。

\section{3 参比气体流量对电动势的影响}

图 5 为参比气体流量由 $10 \mathrm{~mL} / \mathrm{min}$ 增加到 $20 \mathrm{~mL} / \mathrm{min}$, 待电动势稳定后, 又减小回 $10 \mathrm{~mL} / \mathrm{min}$ 的电动势变 化情况。由图 5 可见，当参比气体流量由 $10 \mathrm{~mL} / \mathrm{min}$ 增加到 $20 \mathrm{~mL} / \mathrm{min}$ 时, 传感器的电动势值也由 $0.048 \mathrm{~V}$ 迅速增大到 $0.082 \mathrm{~V}$; 而当气体流量减小回初始状态 时, 电动势值也随即回到原值, 即参比气体流量对 电动势的影响也是一个可逆过程(当流量变成其它 任意值再返回也有此特点)。

将上述两个电动势值代入公式(1)可得铝液氢含 量分别为 0.08 和 $0.05 \mathrm{~mL} / 100 \mathrm{gAl}$, 两者相差 $0.03 \mathrm{~mL} /$ $100 \mathrm{~g} \mathrm{Al}$, 这表明参比气体流量不同会导致传感器 的测氢结果也有所不同。

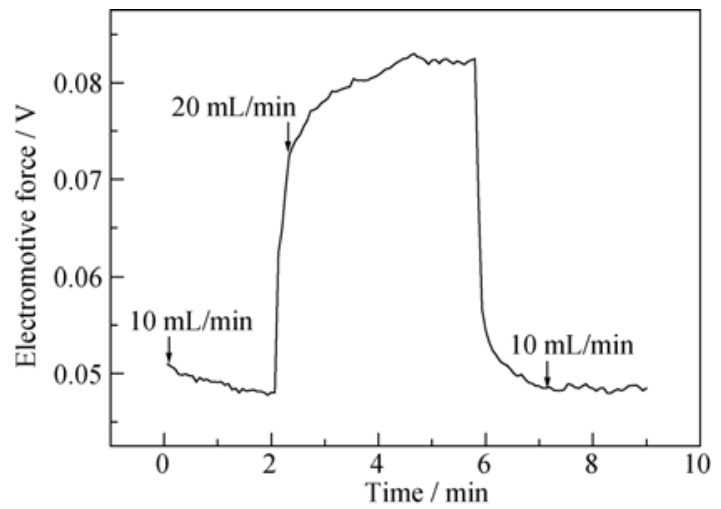

图 5 参比气体流量对传感器电动势的影响

Fig. 5 Dependence of EMF on different gas flow rates 
由流体力学可知, 在管路的气体密度和截面积恒 定条件下, 当参比气体的流量改变时, 气体的压力也 会随之改变, 从而对电动势产生明显影响。若参比气体 的压力或流量选取不适当, 就难以保证实际的氢分压 与公式(1)中的 $P_{\mathrm{H}_{2}}$ (由总压为 1 个大气压计算出氢分压 为 $8.06 \mathrm{mmHg}$ ) 相同, 进而将影响氢含量的准确计算。

通常工业上对气体流量的控制比对压力的控制 更为快捷和准确, 那么在确定参比气体流量时, 就 必须根据气体管路和传感器结构特点找到与计算公 式相匹配的数值, 最好与其它测氢仪进行横向对比, 以实现技术参数的精确确定。

\section{4 参比气体流量对电动势平衡时间的影响}

图 6 为参比气体流量分别为 $10 、 15 、 20 、 25 \mathrm{~mL} / \mathrm{min}$ 时, 电动势达到平衡所需时间的变化情况。由图 6 可知, 随着参比气体流量的增加, 电动势达到平衡所需时间逐 渐延长。这是由于质子导体参比端气体由零流量增大到 设定流量并且达到稳定所需时间逐渐变长导致的。

\section{5 参比气体流量对阻抗谱的影响}

图 7(a)为气体流量 $20 \mathrm{~mL} / \mathrm{min}$ 时测得的传感器电 池阻抗谱, 由图可见, 阻抗谱的形状较有规律, 由两个 变形的容抗圆弧组成, 高频弧表征质子导体的体阻抗, 低频弧表征 Pt 电极和质子导体之间的界面阻抗。利用 等效电路 $\left(R_{\mathrm{c}} Q_{\mathrm{c}}\right)\left(R_{\mathrm{t}} Q_{\mathrm{dl}}\right)$ (图 7(b))对阻抗谱进行拟合处理, 拟合谱与测量谱重合度较好, 拟合方差较小 $\left(6.03 \times 10^{-4}\right)$, 表明此等效电路基本可以反映传感器测氢时的电极过 程。其中, $R_{\mathrm{c}}$ 代表质子在质子导体内部的迁移电阻(由 高频弧在横轴截距获得); $Q_{\mathrm{c}}$ 代表质子导体本身的电容; $Q_{\mathrm{dl}}$ 代表 $\mathrm{Pt} /$ 质子导体界面的双电层电容; $R_{\mathrm{t}}$ 为电荷转移 电阻(由低频弧在横轴截距计算), 它反映质子穿过 $\mathrm{Pt} /$ 质子导体界面进行转移的难易程度。 $R_{\mathrm{t}}$ 的数值愈小, 则质子转移过程愈容易进行。由图可知, $R_{\mathrm{t}}$ 值比 $R_{\mathrm{c}}$ 大, 说明电极反应的速度由电荷转移过程控制。

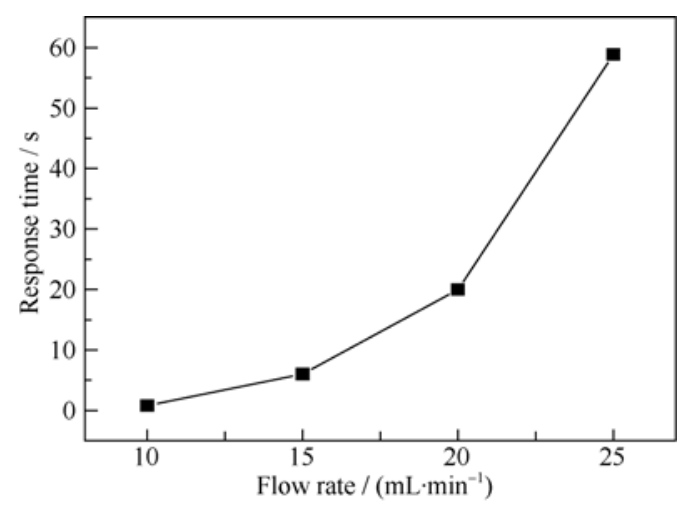

图 6 参比气体流量对电动势平衡时间的影响

Fig. 6 Dependence of equilibrium time of EMF on different gas flow rates

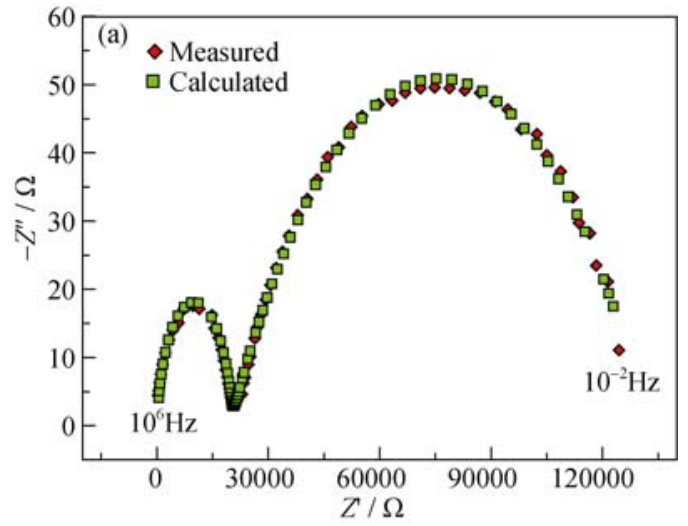

(b)

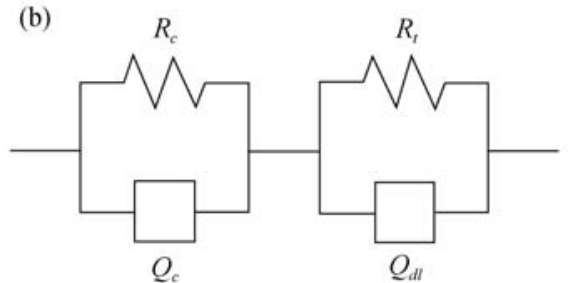

图 7 传感器的阻抗谱 $(\mathrm{a})$ 及其等效电路 $\left(R_{c} Q_{c}\right)\left(R_{t} Q_{d}\right)(\mathrm{b})$

Fig. 7 Impedance spectra (a) and equivalent electrical circuit (b) obtained from hydrogen sensor

( $20 \mathrm{~mL} / \mathrm{min}$ of flow rate of reference gas)

图 8 为参比气瓶输出压力分别为 $0.12 \mathrm{MPa}$ 和 $0.20 \mathrm{MPa}$ 时, 不同气体流量条件下测得的电池阻抗谱。 由图 8 可知, 在参比气瓶输出压力恒定, 气体流量(决定 最后参比气体的压力)逐渐增大条件下，阻抗谱的高频 弧变化不是很明显, 而低频弧有逐渐变小的趋势, 这表 明质子在质子导体内的迁移电阻 $R_{\mathrm{c}}$ 基本相同，但电荷转 移电阻 $R_{\mathrm{t}}$ 逐渐下降, 从而导致体系的总电阻减小。

\section{6 浓差电池型氢洜的脱氢过程}

图 9 为将氢传感器的两根电极引线短接, 使电 池处于短路状态(氢葲), 交替对质子导体管内侧抽 空、输送 $\mathrm{Ar}$ 或 $\mathrm{O}_{2}$ 气进行氢携带时铝液氢含量的变 化曲线。由图 9 中数据计算出, 在短路真空抽取和短 路气体携带联合作用下，铝液中的氢含量由初始的 约 $500 \mathrm{~mL} / 100 \mathrm{~g} \mathrm{Al}$ 最终降至 $6.83 \mathrm{~mL} / 100 \mathrm{~g} \mathrm{Al}$, 脱氢 速度为 $6.36(\mathrm{~mL} / \mathrm{min}) / 100 \mathrm{~g} \mathrm{Al}$, 表明本实验中的浓 差电池型氢洜能够显著脱除铝液中的氢含量, 且脱 氢速度较快。

\section{3 结论}

1) 采用固相法制备了纯度较高、粒度较小的 $\mathrm{CaZr}_{0.9} \mathrm{In}_{0.1} \mathrm{O}_{3-\alpha}$ 质子导体管, 根据浓差电池原理组装 了两种探头形式的氢传感器, 并在 $760^{\circ} \mathrm{C}$ 下进行了铝 液氢含量测定。结果表明, 倒置式探头的电动势曲线 变化较平缓, 约经过 $13 \mathrm{~min}$ 电动势达到较稳定状态; 

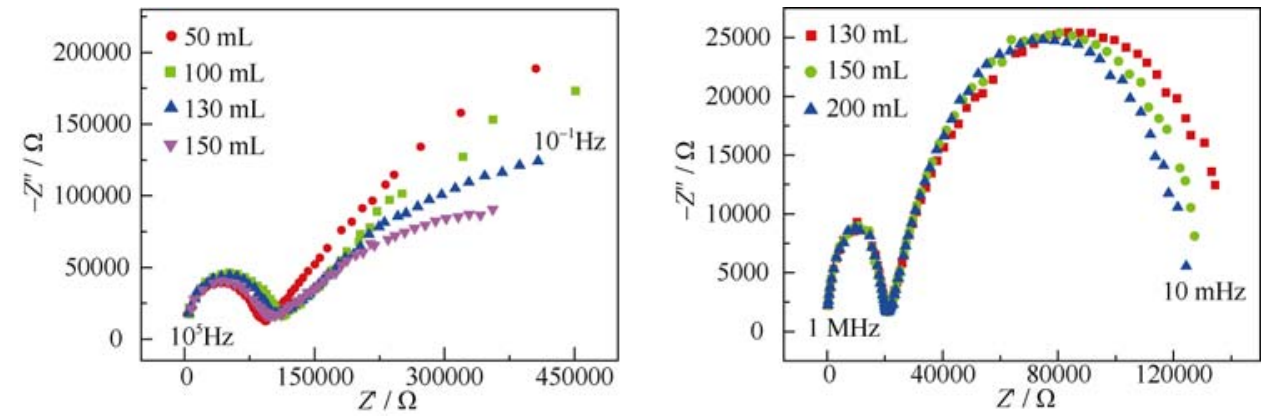

图 8 参比气体流量对阻抗谱的影响

Fig. 8 Dependence of impedance spectra on different gas flow rates Gas cylinder pressure $0.12 \mathrm{MPa}(\mathrm{a})$ and $0.20 \mathrm{MPa}(\mathrm{b})$

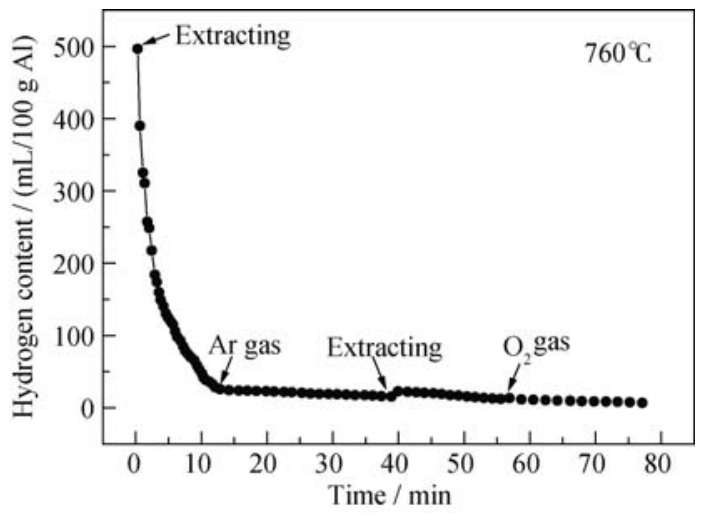

图 9 短路真空抽取或气流携带脱氢法的氢含量曲线

Fig. 9 Relationship between hydrogen content by extracting or gas carrying hydrogen and time at $760^{\circ} \mathrm{C}$

而正置式探头的电动势曲线起伏不平, 难以获得稳定 数值。因此, 倒置式探头的结构设计是比较合理的。

2) 参比气体压力和流量的改变会影响到传感器 参比端的氢分压大小, 从而导致电动势的敏感变化。 其规律是增大参比气体的压力或流量时电动势上升, 反之电动势下降, 是一个可逆过程。此外, 参比气体 流量的增加还会显著延长电动势达到平衡所需的时 间及降低电荷转移电阻。因此, 需根据传感器的气体 管路特点, 选择适当的参比气体流量或压力并进行 精确控制，才能获得快速、准确的测氢结果。

3) 质子导体氢百在短路真空抽取和短路气体 携带辅助作用下, 可将 $760^{\circ} \mathrm{C}$ 铝液中的氢含量由初 始时的 $500 \mathrm{~mL} / 100 \mathrm{~g} \mathrm{Al}$ 降至 $6.83 \mathrm{~mL} / 100 \mathrm{~g} \mathrm{Al}$, 表 明在改善物理条件情况下, 根据浓差电池原理设计 的氢洜可以实现对铝液有效且无污染的脱氢过程, 有较大的研究价值和应用前景。

\section{参考文献:}

[1] IWAHARA H, ESAKA T, UCHIDA H, et al. Proton conduction in sintered oxides and its application to steam electrolysis for hydrogen production. Solid State Ionics, 1981, 3-4: 359-363.

[2] MATSUSHITA E, SASAKI T. Theoretical approach for protonic conduction in perovskite-type oxides. Solid State Ionics, 1999, 125(1-4): 31-37.

[3] 王 东, 范建华, 刘春明, 等. $\mathrm{BaCe}_{1-x} \mathrm{Y}_{x} \mathrm{O}_{3-a}$ 及 $\mathrm{BaCe}_{0.9} \mathrm{Sm}_{0.1} \mathrm{O}_{3-a}$ 质 子导体的表征及组成氢票对铝熔体的脱氢. 金属学报, 2007, 43(17): 1228-1232.

[4] 王 东, 史苍际, 刘春明, 等. $\mathrm{CaZr}_{0.9} \mathrm{In}_{0.1} \mathrm{O}_{3-a}$ 高温质子导体管的 制备及性质表征. 金属学报, 2007, 44(2): 177-182.

[5] XU J H, XIANG J, DING H, et al. Synthesis and electrical properties of $\mathrm{BaCeO}_{3}$-based proton conductors by calcinations of metal-polyvinyl alcohol gel. J. Alloys Compd., 2013, 551: 333-337.

[6] ANANYEV M, MEDVEDEV D, GAVRILYUK A, et al. $\mathrm{Cu}$ and Gd co-doped $\mathrm{BaCeO}_{3}$ proton conductors: experimental vs SEM image algorithmic-segmentation results. Electrochimica Acta, 2014, 125(10): 371-379.

[7] Medvedev D A, GORBOVA E V, DEMIN A K, et al. Conductivity of Gd-doped $\mathrm{BaCeO}_{3}$ protonic conductor in $\mathrm{H}_{2}-\mathrm{H}_{2} \mathrm{O}-\mathrm{O}_{2}$ atmospheres. Int. J. Hydrogen Energy, 2014, 39(36): 21547-21552.

[8] KOKKOFITIS C, OUZOUNIDOU M, SKODRA A, et al. High temperature proton conductors: applications in catalytic processes. Solid State Ionics, 2007, 178(7): 507-513.

[9] SUKSAMAI W, METCALFE I S. Measurement of proton and oxide ion fluxes in a working Y-doped $\mathrm{BaCeO}_{3} \mathrm{SOFC}$. Solid State Ionics, 2007, 178(7-10): 627-634.

[10] STUART P A, UNNO T, KILNER J A, et al. Solid oxide proton conducting steam electrolysers. Solid State Ionics, 2008, 179(21-26): 1120-1124.

[11] ZHANG J C, WEN Z Y, CHI X W, et al. Proton conducting $\mathrm{CaZr}_{0.9} \mathrm{In}_{0.1} \mathrm{O}_{3-\delta}$ ceramic membrane prepared by tape casting. Solid State Ionics, 2012, 225(1): 291-296.

[12] MATSUMOTO H, SHIMURA T, IWAHARA H, et al. Hydrogen separation using proton-conducting perovskites. J. Alloys Compd., 2006, 408-412: 456-462.

[13] XIA T, HE C H, YANG H G, et al. Hydrogen extraction characteristics of high-temperature proton conductor ceramics for hydrogen isotopes purification and recovery. Fusion Engineering \& Design, 2014, 89(7/8): 1500-1504.

[14] NOTORP KYHS-A2 Hydrogen Analyzer for Molten Aluminium Alloys, TYK Corporation, Tokyo, Japan, 1997.

[15] FUKATSU N, KURITA N, OHASHI T. Hydrogen Sensor for Molten Metals and Its Application to Materials Processing. Second International Conference on Properties on Processing Materials for Properties. Pennsylvania, 2000: 357-363. 